OPEN ACCESS

Edited by:

Brian Silver,

University of Massachusetts

Medical School, USA

Reviewed by:

Marc Malkoff,

University of Tennessee Health

Sciences Center, USA

Henry Ma,

Monash University, Australia

*Correspondence: Yong Jiang

jiangyong@swmu.edu.cn

Specialty section: This article was submitted to Stroke, a section of the journal

Frontiers in Neurology

Received: 10 December 2016 Accepted: 31 March 2017 Published: 19 April 2017

Citation:

Peng J-h, Qin $X-h$, Pang J-w, Wu Y,

Dong J-h, Huang $C-r$, Wan $W-f$, Yang $X-b$, Sun $X-c$, Chen $L-g$ and Jiang $Y$ (2017) Apolipoprotein E $\varepsilon 4$ : A Possible Risk Factor of Intracranial Pressure and White Matter Perfusion in Good-Grade Aneurysmal Subarachnoid Hemorrhage Patients at Early Stage. Front. Neurol. 8:150. doi: 10.3389/fneur.2017.00150

\section{Apolipoprotein E ع4: A Possible Risk Factor of Intracranial Pressure and White Matter Perfusion in Good-Grade Aneurysmal Subarachnoid Hemorrhage Patients at Early Stage}

Jian-hua Peng1, Xing-hu Qin',2, Jin-wei Pang1, Yue Wu ${ }^{3}$, Jin-hu Dong ${ }^{1}$, Chang-ren Huang ${ }^{1}$, Wei-feng Wan', Xiao-bo Yang ${ }^{1}$, Xiao-chuan Sun ${ }^{3}$, Li-gang Chen ${ }^{1}$ and Yong Jiang ${ }^{1 *}$

'Department of Neurosurgery, The Affiliated Hospital of Southwest Medical University, Luzhou, China, ${ }^{2}$ Department of Neurosurgery, People's Hospital of Deyang City, Deyang, China, ${ }^{3}$ Department of Neurosurgery, The First Affiliated Hospital of Chongqing Medical University, Chongqing, China

Aneurysmal subarachnoid hemorrhage (aSAH) is a devastating and complicated disease with significant morbidity and mortality. Previous studies have shown that genetic susceptibility may play an important role in the outcome of a given individual with aSAH. This study evaluates the potential association in effects of the APOE allele on the early brain injury (EBI) in light of elevated intracranial pressure (ICP) and cerebral perfusion disorders in a consecutive series of non-comatose Chinese patients with aSAH. A total of 122 patients with aSAH (54 males and 68 females) were enrolled in this study. Demographic and clinical data were collected. We measured ICP before microsurgical clipping or endovascular coiling during the first $72 \mathrm{~h}$ after aneurysm rupture. Computed tomography perfusion (CTP) examination in patients was performed before treatment. The distributions of APOE genotypes and alleles matched Hardy-Weinberg law $(p>0.05)$. In this study, 68 patients (55.7\%) had a normal ICP, whereas 54 (44.3\%) had an elevated ICP. Fourteen of 21 patients with APOE $\varepsilon 4$ had an elevated ICP, which was significantly different from those without APOE $\varepsilon 4(p=0.03)$. The patients with the $\varepsilon 4$ allele had a higher incidence of elevated ICP $[p=0.009$, 95\% confidence interval $(C l)=1.481-15.432$, odds ratio $=4.780$ ] than those without this allele. For CTP measurements, a lower mean cerebral blood flow (difference, $-4.74 ; 95 \% \mathrm{Cl}$, 0.53-8.94 s, $p=0.03$ ), longer mean transit time (difference, $0.47 ; 95 \% \mathrm{Cl},-0.87$ to $-0.78, p=0.02$ ), and time-to-peak (difference, 2.29; $95 \% \mathrm{Cl},-3.64$ to $-0.93 \mathrm{~s}, p=0.02$ ) were observed in patients with $\varepsilon 4$ allele than in those without in the internal capsule regions. In conclusion, the APOE $\varepsilon 4$ allele predisposes patients to elevated ICP and perfusion disorders in white matter regions during the first $72 \mathrm{~h}$ after aSAH. The presence of an APOE $\varepsilon 4$ allele plays an important role in the $\mathrm{EBI}$ response to aSAH.

Keywords: apolipoprotein E, subarachnoid hemorrhage, early brain injury, intracranial pressure, computed tomography perfusion, white matter injury 


\section{INTRODUCTION}

Aneurysmal subarachnoid hemorrhage (aSAH) is a devastating and complicated disease with significant morbidity and mortality (1). Traditionally, cerebral vasospasm (CVS) and aneurismal rebleeding have been considered to be major complications that significantly worsen the prognosis of aSAH (2). In recent years, early brain injury (EBI) during the first $72 \mathrm{~h}$ after aSAH has been shown to play an important determinant of clinical outcome (3). Notably, global cerebral ischemia as a hallmark of EBI is thought to be related to raised intracranial pressure (ICP) and cerebral perfusion (4-6). Cerebral perfusion is related to mean arterial blood pressure and ICP, which reduces the cerebral perfusion and threatens the brain function.

Increasing evidence has shown that aSAHs with similar pathologies might have different clinical statuses and outcomes. However, the Hunt and Hess grade, sex, and age of the patient can explain only part of this variability. Alternatively, genetic susceptibility may play an important role in the outcome of a given individual with aSAH $(7,8)$. Recently, apolipoprotein E $(\mathrm{APOE}=$ gene; apoE $=$ protein $)$ has become one of the most widely studied genes in aSAH research (9). There are three common isoforms (apoE2, apoE3, and apoE4) encoded by three gene alleles (APOE $\varepsilon 2, \mathrm{APOE} \varepsilon 3$, and APOE $\varepsilon 4$ ) (10). Although the presence of the APOE $\varepsilon 4$ allele predisposes poorer outcome in patients with aSAH, the conclusions of investigations are still conflicting $(11,12)$.

Increased ICP is common after SAH, even in patients with a good clinical $\mathrm{H}-\mathrm{H}$ grade. Elevated ICP post-SAH is associated with a worse patient outcome (13). Patients with good-grade aSAH (Hunt and Hess grades I-III) can also die or present severe deficit due to increased ICP leading to cerebral ischemia (14). Elevated ICP post-SAH is associated with good-grade patient outcomes (13). Our previous study indicated that APOE\&4 as an independent risk factor for cerebral perfusion dysfunction (6). However, the risk of APOE genotype on ICP and cerebral perfusion of good-grade aSAH patients has never been investigated in detail. The present study aimed to evaluate the potential association between APOE allele and the progression of EBI in light of increased ICP and cerebral perfusion in a consecutive series of good-grade Chinese patients with aSAH.

\section{MATERIALS AND METHODS}

\section{Study Design and Inclusion and Exclusion Criteria}

A total of 122 consecutive patients with onset of aSAH shorter than $72 \mathrm{~h}$ were enrolled to the two departments of neurosurgery in this prospective pilot study from December 2014 to July 2016 (The Affiliated Hospital of Southwest Medical University: 67 patients and The First Affiliated Hospital of Chongqing Medical University: 55 patients). aSAH was confirmed using CT scans and lumbar puncture. The sample group of adult patients included 54 males and 68 females, aged 48-72 years old, with a diagnosis of SAH from a ruptured cerebral aneurysm, which was verified using digital subtraction angiography or CT angiography.
The following inclusion criteria were applied: (i) the clinical grade before treatment was I-III, according to the Hunt and Hess grading system (e.g., good-grade non-comatose patients); (ii) all of the patients were treated within $72 \mathrm{~h}$ with early microsurgical clipping; and (iii) clinical data were recorded completely.

We excluded patients with the following characteristics: (i) cerebral lesions other than aneurysms because they can be independent predictors of ICP increases and can render the attribution of ICP less reliable; (ii) displayed symptomatic CVS with a $P M V \geq 120 \mathrm{~cm} / \mathrm{s}$ (15) or severe narrowing of the involved artery on cerebral angiogram within the first $72 \mathrm{~h}$; and (iii) aneurysmal rebleeding, intra-ventricular hemorrhage, or hydrocephalus observed by CT scan within $72 \mathrm{~h}$.

\section{Recording of Clinical Information}

The clinical data of the included patients were recorded; this information included the patient's age, sex, neurological condition before treatment (Hunt-Hess grade), and Fisher grade on admission as well as the site of the ruptured aneurysm, and the type of treatment (clipping or coiling).

\section{APOE Genotype}

APOE genotype was determined from genomic DNA extracted from venous blood that was collected from the patients upon admission. DNA was extracted from frozen blood using standard techniques, and $\mathrm{APOE} \varepsilon 2, \varepsilon 3$, and $\varepsilon 4$ genotyping was performed using the polymerase chain reaction-restriction fragment length polymorphism method, as previously described (16). Briefly, a 250-bp fragment containing the coding region was amplified. The following primers were used: P1: 5'-TAAGCTTGGCACGGCTGTCCAAGGA-3' (upstream) and P2: 5' -ACAGAATTCGCCCCGGCCTGGTACAC-3' (downstream). The PCR products were digested using the HhaI restriction enzyme, and the fragments were separated by electrophoresis on $4 \%$ ethidium bromide-containing agarose gels for genotype determination.

\section{ICP Monitoring}

Lumbar puncture allowed us to gage the ICP of the SAH patients (17). Thus, lumbar pressure (LP) was used in this study as a surrogate measurement of ICP. After the source of SAH was identified, either aneurysm clipping or coiling was performed within $72 \mathrm{~h}$. Before the operation, a silicon lumbar catheter was introduced through a $16-\mathrm{G}$ lumbar needle into the subarachnoid space at the L4-L5 level. This catheter was connected to a sterile collecting system fixed above the point of catheter insertion. The LP was measured via a hydrostatic pressure transducer, which was immediately connected to the drainage system. The zero reference pressure was the atmospheric pressure at the level of the foramen of Monro. The ICP threshold was set at $20 \mathrm{mmHg}$ because this pressure is the recommended threshold for routine initiation of ICP treatment in neurosurgery in adults.

\section{Computed Tomography Perfusion (CTP) Screening}

To characterize the region-specific response to cerebral perfusion, CTP examination in patients was performed before treatment. After injection $30 \mathrm{ml}$ of non-ionic contrast agent (iopromide 
Ultravist, $370 \mathrm{mg}$ iodine/ml; Schering, Berlin, Germany) and $15 \mathrm{ml}$ of normal saline through the cubital vein $(4 \mathrm{ml} / \mathrm{s})$, head scanning was performed by a Lightspeed VCT 64-slice CT machine. The following parameters were used: $80 \mathrm{kVp}, 200 \mathrm{mAs}$, $5 \mathrm{~mm}$ slice thickness. Original data were transferred to an Adw4.2 workstation for subsequent analysis. The measurements of arterial input (anterior cerebral artery) and venous output (superior sagittal sinus) functions were semiautomatic. We cooperated with the clinical radiologists because they will likely become expert in distinguishing CT images on a region-by-region basis. Hand-drawn, multi regions of interest (ROIs) were defined by prespecified anatomical boundaries on CT images. One side and the contralateral mirror area of caudate nucleus, internal capsule, thalamus, external capsule, and whole cortex were scanned as the ROI. The cerebral blood volume, cerebral blood flow (CBF), mean transit time (MTT), and time-to-peak (TTP) were obtained. To ensure the accuration of each ROI, we narrowed ROIs in regions with indistinguishable boundary, and all the ROIs of each patient were drawn by the same assessor.

\section{Statistical Analysis}

SPSS software (version 19.0) was used for statistical analyses. After counting the number of alleles, the allele frequencies in aSAH patients were calculated from the sample proportions. The constituent ratio of each correlation factor in the clinical data between the groups (with $\varepsilon 4$ allele and without $\varepsilon 4$ allele) was analyzed using the $\chi^{2}$ test for nominal variables. Univariate logistic regression was performed to analyze the associations of age ( $\leq 65$ years old vs. $>65$ years old), Hunt-Hess grade ( 1 and 2 vs. 3), Fisher grade (1 and 2 vs. 3 and 4), aneurysm site (anterior communicating artery vs. other sites), type of treatment (clipping vs. coiling), and APOE genotype (with $\varepsilon 4$ vs. without $\varepsilon 4$ ) with elevated ICP. After adjusting the clinical data, further multivariate logistic regression analyses (stepwise backward conditional and Wald functions, as appropriate) were used to analyze the associations of the APOE genotype (with $\varepsilon 4$ vs. without $\varepsilon 4$ ) with elevated ICP. Variables entered into the multivariate regression at $p<0.05$, while some variables were removed at $p>0.1$. Adjusted odds ratios (ORs) and 95\% confidence intervals (CIs) were calculated from the logistic regression model coefficients. In addition, we compared differences in mean values of cerebral perfusion between patients with and without $\varepsilon 4$ allele by calculating the 95\% CIs of the mean difference. The statistical significance of the correlation between the investigated quantitative variables was determined by the $p$ value, and a $p$ value $<0.05$ was considered significant.

\section{RESULTS}

The department admitted 210 patients with aSAH during the study period. Seventy-one patients were not included in the study because their clinical conditions were classified as IV or $\mathrm{V}$ on the Hunt and Hess grading scale. Seventeen good-grade patients met the exclusion criteria and were disqualified: two patients had cerebral lesions other than aneurysms, six patients had aneurysmal rebleeding or hydrocephalus within $72 \mathrm{~h}$, three patients had intracerebral hematomas, and six patients displayed symptomatic CVS, with a PMV $\geq 120 \mathrm{~cm} / \mathrm{s}$, or suffered from severe narrowing of the involved artery as observed on cerebral angiography within $72 \mathrm{~h}$. The remaining $122 \mathrm{aSAH}$ patients were enrolled in the current study.

Of the 122 patients, allele frequencies were $9.4 \%$ for the $\varepsilon 4$ allele and $90.6 \%$ for the non- $\varepsilon 4$ allele. The distributions of the APOE allele frequencies and genotypes are presented in Table 1. The samples demonstrated Hardy-Weinberg equilibrium $(p>0.05)$.

The clinical data of the included patients are summarized in Table 2; these data include age, sex, Hunt and Hess grade and Fisher grade of CT scans, aneurysm site, and the type of treatment. The distribution of the baseline characteristics between the groups of patients with and without an APOE $\varepsilon 4$ allele was not different $(p>0.05)$.

\section{Effect of APOE $\varepsilon 4$ Allele on ICP}

All of the ICP analyses were based on values obtained from lumbar drainage. The patients were separated into two groups based on their LP values before treatment. ICP values of $20 \mathrm{mmHg}$ were chosen because an ICP greater than $20 \mathrm{mmHg}$ is a well-known threshold for treating patients with neurosurgery. Therefore, the normal ICP group included patients who had an ICP less than $20 \mathrm{mmHg}$, and the elevated ICP group consisted of patients with an ICP greater than $20 \mathrm{mmHg}$. In this study, 68 patients (55.7\%) had a normal ICP, whereas $54(44.3 \%)$ had an elevated ICP. The ICP was increased in good-grade patients with a Hunt and Hess grade of I-III. Fourteen of 21 patients with APOE $\varepsilon 4$

TABLE 1 | Distribution of APOE genotype.
\begin{tabular}{cccccccccc}
\multicolumn{1}{c}{ Genotype } \\
\hline$\varepsilon 2 / 2$ & $\varepsilon 2 / 3$ & $\varepsilon 2 / 4$ & $\varepsilon 3 / 3$ & $\varepsilon 3 / 4$ & $\varepsilon 4 / 4$ & $\varepsilon 2$ & $\varepsilon 3$ & $\varepsilon 4$ \\
0 & 15 & 4 & 86 & 15 & 2 & $7.8 \%$ & $82.8 \%$ & $9.4 \%$ \\
\hline
\end{tabular}

TABLE 2 | Baseline characteristics of patients with and without $\varepsilon 4$ allele.

\begin{tabular}{|c|c|c|c|}
\hline & $\begin{array}{l}\text { Total series } \\
(n=122)\end{array}$ & $\begin{array}{l}\text { Patients with } \varepsilon 4 \\
\text { allele }(n=21)\end{array}$ & $\begin{array}{l}\text { Patients without } \varepsilon 4 \\
\text { allele }(n=101)\end{array}$ \\
\hline \multicolumn{4}{|l|}{ Age } \\
\hline$\leq 65$ years & 80 & 10 & 70 \\
\hline$>65$ years & 42 & 11 & 31 \\
\hline \multicolumn{4}{|l|}{ Sex } \\
\hline Male & 54 & 9 & 45 \\
\hline Female & 68 & 12 & 56 \\
\hline \multicolumn{4}{|c|}{$\mathrm{H}-\mathrm{H}$ grade } \\
\hline 1 and 2 & 64 & 11 & 53 \\
\hline 3 & 58 & 10 & 48 \\
\hline \multicolumn{4}{|c|}{ Fisher grade } \\
\hline 1 and 2 & 53 & 13 & 40 \\
\hline 3 & 69 & 8 & 61 \\
\hline \multicolumn{4}{|l|}{ Site } \\
\hline AcoA & 50 & 9 & 41 \\
\hline Other & 72 & 12 & 60 \\
\hline \multicolumn{4}{|l|}{ Treatment } \\
\hline Clipping & 98 & 16 & 81 \\
\hline Coiling & 24 & 5 & 19 \\
\hline
\end{tabular}


had an elevated ICP, which was significantly different from those without $\mathrm{APOE} \varepsilon 4(p=0.03)$. According to the univariate analysis, patients with the $\varepsilon 4$ allele had a higher incidence of elevated ICP than those without this allele $(p=0.03,95 \% \mathrm{CI}=1.132-8.217$, $\mathrm{OR}=3.1$ ). When the data were adjusted for significant risk factors in the multivariate analysis (age, sex, Hunt and Hess grade, Fisher grade, etc.), the association of the $\varepsilon 4$ allele with the risk of elevated ICP was even more significant $(p=0.009,95 \% \mathrm{CI}=1.481-15.432$, $\mathrm{OR}=4.780)$. Furthermore, elevated ICP was also associated with a worse Hunt-Hess grade $(p=0.004,95 \% \mathrm{CI}=1.536-9.116$, $\mathrm{OR}=3.742$ ) (Table 3 ). The patient's age, sex, Fisher grade, and aneurysm site were not statistically associated with ICP.

\section{Assessment of the APOE $\varepsilon 4$ Allele and Cerebral Perfusion}

As shown in Table 4, in the internal capsule area, CBF was $33.03 \mathrm{~mL} / 100 \mathrm{~g} / \mathrm{min}$ in patients with $\varepsilon 4$ allele and $37.77 \mathrm{~mL} /$ $100 \mathrm{~g} / \mathrm{min}$ in those without $\varepsilon 4$ allele (difference, $-4.74 ; 95 \% \mathrm{CI}$, $0.53-8.94 \mathrm{~s}, p=0.03)$. In patients with APOE $\varepsilon 4$, MTT was $6.29 \mathrm{~s}$, and in those without $\varepsilon 4$ allele, MTT was $5.33 \mathrm{~s}$ in internal capsule (difference, $0.47 ; 95 \% \mathrm{CI},-0.87$ to $-0.78 \mathrm{~s}, p=0.02$ ). As was TTP, patients with $\varepsilon 4$ allele had a significantly longer TTP than patients without $\varepsilon 4$ allele both in internal capsule (difference, 2.29; 95\% $\mathrm{CI},-3.64$ to $-0.93 \mathrm{~s}, p=0.02$ ) and external capsule (difference, $1.09 ; 95 \% \mathrm{CI},-2.16$ to $-0.01 \mathrm{~s}, p=0.04$ ). These results suggested that patients with $\varepsilon 4$ allele had a significant abnormality in brain cerebral perfusion in internal capsule and other whiter matter regions, such as external capsule (Figure 1).

\section{DISCUSSION}

From the study of 122 good-grade aSAH patients, our major finding is that those aSAH patients carrying the APOE $\varepsilon 4$ allele are predisposed to elevate ICP within $72 \mathrm{~h}$ of the ictus, after controlling for age, the severity of the hemorrhage, the aneurysm location, and other factors. Moreover, patients with $\varepsilon 4$ allele had a significant abnormality in brain cerebral perfusion in white matter (especially in the internal capsule area). The current study provides some initial clinical evidence that the APOE $\varepsilon 4$ allele is associated with a detrimental effect on a patient's early response to aSAH.

Recently, particular attention has been paid to the APOE gene. apoE is associated with complex neuroprotective functions in the biochemical network of SAH $(10,18,19)$. However, the neuroprotective effectiveness of the apoE4 isoform is reduced when compared with the other isoforms, and this decreased effectiveness has designated the $\varepsilon 4$ allele as a sort of "frailty protein" and a potential risk factor. Several possible mechanisms might be involved in different ways $(10,18)$ : (i) apoE4 might be a defective free radical scavenger; (ii) patients with the apoE4 protein could have impaired modulation of the brain inflammatory response to SAH, due to unregulated cytokine cascades; or (iii) reduced membrane repair and synaptic plasticity might occur.

Although controversial, increasing evidence indicates that the APOE $\varepsilon 4$ genotype predisposes the patient to a poor outcome following aSAH $(11,12)$. In the Chinese population, the $\varepsilon 4$ allele is also thought to be a possible risk factor for poor outcomes following aSAH (20). In our previous work, a series of follow-up clinical studies also demonstrated that patients with the APOE $\varepsilon 4$ allele were prone to CVS and brain dysfunction, instead of rebleeding, after spontaneous $\mathrm{SAH}$, which can also contribute to poor outcomes $(21,22)$.

In aSAH patients, global hypoperfusion caused by increased ICP and focal ischemia induced by vasospasm are common and devastating outcomes. In recent decades, CVS has become the focus of a large number of clinical and experimental research efforts. Although vasospasm is regarded as the primary cause of mortality and neurological morbidity in patients initially surviving aSAH, therapies designed to prevent and treat vasospasm remain limited $(4,23)$. Increasing evidence has led researchers to cast doubt on the importance of vasospasm in the setting of $\mathrm{SAH}$. In recent years, EBI has been suggested to play a key role in aSAH patients' clinical deterioration and subsequent poor outcomes.

A previous study conducted on non-comatose patients with aSAH found that the presence of the $\varepsilon 4$ allele increased the risk of clinical vasospasm, enhanced cognitive morbidity, and delayed ischemic neurologic deficit recovery (24). As increased ICP is common after aSAH, even in patients with good clinical grades, outcomes are negatively correlated with increased ICP. ICP appears to be a stronger predictor than the influence of vasospasm (25). To our knowledge, this relationship has not been reported in the clinical studies exploring the APOE gene's influence on ICP in the early stages of aSAH. In the present study, we measured ICP using lumbar drainage before surgery as a surrogate measurement, and we demonstrated that patients with the APOE $\varepsilon 4$ polymorphism

TABLE 3 | Intracranial pressure logistic regression.

\begin{tabular}{|c|c|c|c|c|c|c|}
\hline \multirow[t]{2}{*}{ Characteristic } & \multicolumn{3}{|c|}{ Univariate } & \multicolumn{3}{|c|}{ Multivariate } \\
\hline & $p$ Value & OR & $95 \% \mathrm{Cl}$ & $p$ Value & OR & $95 \% \mathrm{Cl}$ \\
\hline APOE $\varepsilon 4$ & $0.027^{\star}$ & 3.1 & $1.132-8.217$ & $0.009^{\star}$ & 4.780 & $1.481-15.432$ \\
\hline Sex & 0.256 & 0.7 & $0.320-1.355$ & 0.438 & 0.717 & $0.309-1.664$ \\
\hline Age & 0.821 & 0.9 & $0.432-1.947$ & 0.416 & 0.669 & $0.254-1.762$ \\
\hline $\mathrm{H}-\mathrm{H}$ grade & $0.022^{*}$ & 2.4 & $1.132-4.879$ & $0.004^{\star}$ & 3.742 & $1.536-9.116$ \\
\hline Fisher grade & 0.866 & 1.1 & $0.517-2.189$ & 0.133 & 2.013 & 0.809-5.008 \\
\hline Site & 0.247 & 1.5 & $0.740-3.218$ & 0.022 & 3.013 & $1.176-7.717$ \\
\hline
\end{tabular}

OR, odds ratio; $\mathrm{Cl}$, confidence interval.

*Significant difference $(p<0.05)$. 


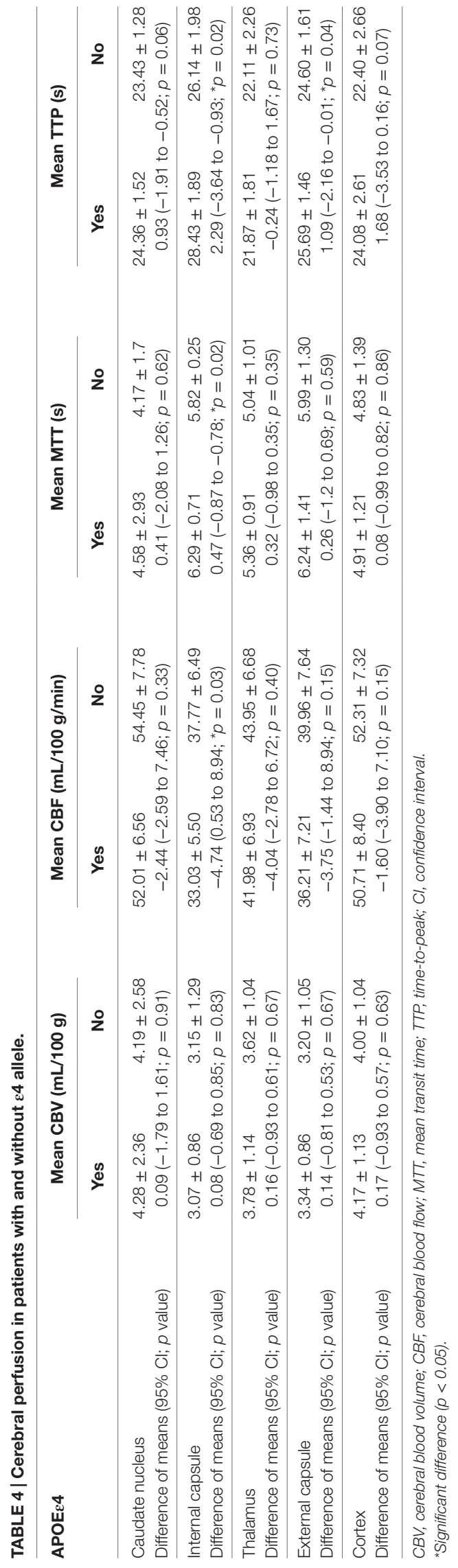

were predisposed to the presence of elevated ICP during the early phases of aSAH. In one TBI study, APOE 4 could increase cellular and tissular vulnerability (26), Olivecrona and coworkers found that there was no statistically significant difference in the ICP or cerebral perfusion pressure (CPP) of $\varepsilon 4$ and non- $\varepsilon 4$ patients with severe TBI who were treated with an ICP-targeted therapy (24). These results are apparently in contrast with those of our study. However, the two reports are not actually comparable because of their different neurological diseases, inclusion/exclusion criteria, and so on.

Early brain injury is believed to arise from significant pathophysiological mechanisms that occur in the brain during aSAH $(2,3)$. The acute rise in ICP, which has been demonstrated in both clinical studies and experimental animal models, results from the initial mass effect of the blood flowing into the subarachnoid space $(27,28)$, impeded CSF drainage (29), cerebrovascular dysfunction (30), and the development of cerebral edema (31). Elevated ICP may be correlated with the extent of EBI (32). As the pressure rises, a compensatory decrease in CPP occurs. The mechanism behind this relationship may be related to the Monroe-Kelly hypothesis. If the compensatory mechanism is not sufficient to support critical levels of brain perfusion, increased ICP and subsequently decreased CPP can result in significant reductions in $\mathrm{CBF}$, leading to the deterioration of neurological function. apoE4 exerts a strong influence of cerebrovasculature dysfunction and disrupts CBF (33). In our previous study, APOEع4 carriers exhibit MTT prolongation in CTP scanning within $24 \mathrm{~h}$ (6). We further measured the region-specific response to cerebral perfusion in this study. We found a significant prolongation of MTT and TTP in the internal capsule and TTP in the external capsule of patients with $\varepsilon 4$ allele. Although the reduction of values are of small differences, the $\varepsilon 4 \mathrm{aSAH}$ patients presented a lower $\mathrm{CBF}$ in regions measured in this study, while the internal capsule region presented a significant difference. Furthermore, the MTT prolonged over $5.9 \mathrm{~s}$ in some regions, such as internal capsule. Our previous study indicated APOE\&4 as an independent risk factor for prolonged MTT over $5.9 \mathrm{~s}$, suggestive of increased risk of DCI and poor outcome in APOE\&4 patients (6). Meanwhile, a significant reduction of $\mathrm{CBF}$ was seen in internal capsule of patients with $\varepsilon 4$ allele. Previous study demonstrates that astrocytes are capable of eliciting both vasoconstriction and vasodilation, then control of CBF in brain (34). The apoE4-induced detrimental changes may be linked to astrocyte activation (33). Although several limitations of CTP should be considered, this technology may reflect the white matter injury indirectly according to regional perfusion. The abnormality in brain cerebral perfusion in white matter regions suggested that patients with $\varepsilon 4$ allele might suffer more serious white matter injury after aSAH.

White matter injury has recently been reported in clinical and experimental SAH $(35,36)$. Victims of SAH suffer most from global emotional and cognitive dysfunction and associated diffuse cerebral atrophy, and diffuse axonal injury may also be an important feature of SAH $(37,38)$. van Asch and coworkers also observed an abnormalities perfusion of white matter in acute hydrocephalus aSAH patients (39). Studies support the hypothesis that white matter injury is due to CVSs and perfusion disorders after a brain injury (40-42). Although this has been 


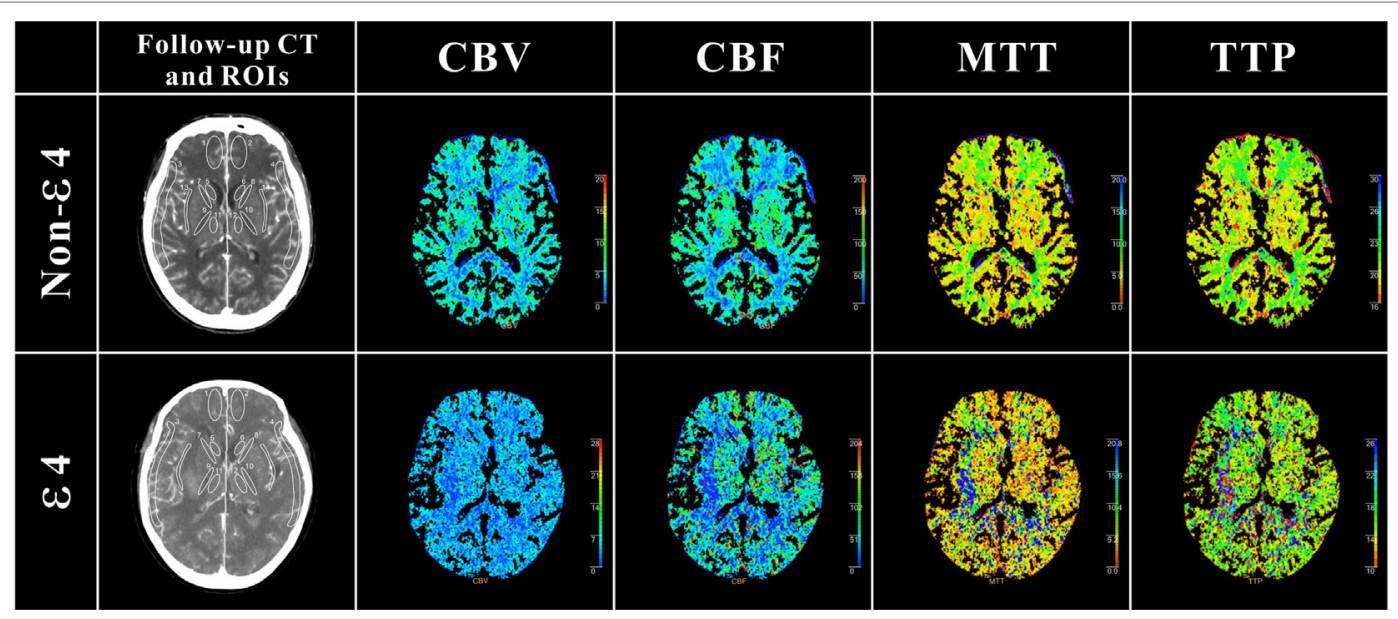

FIGURE 1 | Definition of regions of interest (ROIs) in follow-up CT and cerebral perfusion images in $\varepsilon 4$ and non- $\varepsilon 4$ SAH patients. ROls were drawn in 5 different regions: cortex: ROls 1 and 2 (anterior cerebral artery), ROls 3 and 4 (middle cerebral artery); caudate nucleus: ROls 5 and 6 ; internal capsule: ROls 7 and 8 (anterior limb), ROls 9 and 10 (posterior limb); thalamus external: ROls 11 and 12; capsule: ROls 13 and 14. As shown in cerebral perfusion images, APOE $\varepsilon 4$ allele aneurysmal subarachnoid hemorrhage patients suffered more severe perfusion disorders, especially in white matter regions.

identified as the only proven drug to improve patients' outcomes after SAH, nimodipine, a kind of calcium antagonist, needed to advance the understanding of its effects on microcirculatory changes and cerebral perfusion after SAH. Thus, development of feasible SAH therapeutic strategies still needs more effort. Our previous study has identified ApoE-mimetic peptide COG1410 in EBI by preventing white matter injury after experimental SAH (43). Because ApoE-mimetic peptide is a modified peptide sequence from human apoE, this could indicate that apoE4 lacks a protective effect found in apoE2 and apoE3 in the biochemical network of SAH. Hence, APOE 4 may induce cerebral perfusion impairment via elevated ICP in the early phase, contributing to white matter injury in EBI following aSAH. Furthermore, increased postoperative ICP is common after early clipping, especially in poor-grade aSAH patients, and this might precipitate CVS-induced ischemic events in aSAH patients including the deterioration of neurological function and poor outcomes (44, 45). Patients with symptomatic CVS were excluded in this study, the results indicated that one of the most important factors in predicting the outcomes is correlated with elevated ICP, including good $\mathrm{H}-\mathrm{H}$ grade aSAH patients with $\varepsilon 4$ allele.

Although no previous clinical studies have specifically focused their attention on ICP after SAH, these results are supported by the experimental observations that wild-type mice treated with ApoE-mimetic peptide after experimental SAH decreased mortality, functional deficits and vasospasm (25), neurons apoptosis (46), and BBB disruption (47) as compared with vehicle-treated mice. Notably, in that study, SAH-affected mice expressing the human $\varepsilon 4$ allele were prone to higher mortality rates and greater functional deficits compared to their human $\varepsilon 3$ counterparts during the first $72 \mathrm{~h}$ after experimental SAH. These deficits were associated with a greater degree of cerebral edema and vasospasm in mice expressing the apoE4 isoform. Modulation of the CNS inflammatory response might be one mechanism by which APOE genotype affects EBI after SAH.

\section{Limitations}

There were several potential limitations of the current study. First, as blood is mainly located in the basal cisterns in aSAH patients, CSF outflow from the ventricles into the subarachnoid space should not be substantially impaired. At the same time, the location of the blood clot can present a barrier to the downstream flow of CSF from the convex subarachnoid space of the cerebral hemispheres through the foramen magnum into the spinal compartment. Thus, a partial obstruction might be present and render the LP lower than the actual ICP. Second, although lumbar drainage has been shown to be safe in SAH patients, the formation of pressure gradients remains a potential threat that could result in herniation and rebleeding. Therefore, lumbar drainage was only performed in the present study before aneurysm clipping or coiling. An additional method, such as continuous ICP monitoring, might be needed to verify our findings. Third, abnormality in brain cerebral white matter perfusion of $\varepsilon 4$ allele aSAH patients was observed in this study. However, the specific reason of white matter injury after SAH is not entirely clear. The detail pathophysiological processes and clinical diagnosis strategy of white matter injury after SAH need to be further investigated both in experimental and clinical studies.

\section{Conclusion and Outlook}

We evaluated the association between the presence of the APOE $\varepsilon 4$ allele and elevated ICP via invasive (LP) in this prospective study. Despite the limitations of the present study, these preliminary findings provide new evidence that the APOE $\varepsilon 4$ allele predisposes patients to elevated ICP after aSAH. Meanwhile, APOE $\varepsilon 4$ allele aSAH patients suffered more severe perfusion disorders in white matter regions. These clinical observations support the hypothesis that APOE plays an important role in the response of the CNS to EBI, possibly involving the adverse effects of apoE4 on white matter injury, neurobiology, and its potential links to certain risk factors, such as elevated ICP. To improve the 
precision medicine, further studies are needed to elucidate the mechanisms by which the APOE $\varepsilon 4$ allele can adversely affect a patient's response to EBI after aSAH.

\section{ETHICS STATEMENT}

The study was approved by the ethics committee of the coordinating institution and has therefore been performed in accordance with the ethical standards laid down in the 1964 Declaration of Helsinki and its later amendments. All the patients gave their informed consent prior to their inclusion in the study. Informed consent was obtained from each patient directly.

\section{AUTHOR CONTRIBUTIONS}

Conception and design: YJ and J-hP. Acquisition of data: J-wP, $\mathrm{X}-\mathrm{hQ}$, and X-bY. Analysis and interpretation of data: YW, J-hD, W-fW, and C-rH. Drafting the article: J-hP and YJ. Reviewed

\section{REFERENCES}

1. Suarez JI, Tarr RW, Selman WR. Aneurysmal subarachnoid hemorrhage. N Engl J Med (2006) 354:387-96. doi:10.1056/NEJMra052732

2. Sehba FA, Hou J, Pluta RM, Zhang JH. The importance of early brain injury after subarachnoid hemorrhage. Prog Neurobiol (2012) 97:14-37. doi:10.1016/ j.pneurobio.2012.02.003

3. Caner B, Hou J, Altay O, Fujii M, Zhang JH. Transition of research focus from vasospasm to early brain injury after subarachnoid hemorrhage. J Neurochem (2012) 123(Suppl 2):12-21. doi:10.1111/j.1471-4159.2012.07939.x

4. Voldby B, Enevoldsen EM. Intracranial pressure changes following aneurysm rupture. Part 1: clinical and angiographic correlations. J Neurosurg (1982) 56:186-96. doi:10.3171/jns.1982.56.2.0186

5. Marbacher S, Andereggen L, Neuschmelting V, Widmer HR, Von Gunten M, Takala J, et al. A new rabbit model for the study of early brain injury after subarachnoid hemorrhage. J Neurosci Methods (2012) 208:138-45. doi:10.1016/j. jneumeth.2012.05.010

6. Cheng C, Jiang L, Yang Y, Wu H, Huang Z, Sun X. Effect of APOE gene polymorphism on early cerebral perfusion after aneurysmal subarachnoid hemorrhage. Transl Stroke Res (2015) 6(6):446-50. doi:10.1007/s12975-015-0426-6

7. Mccolgan $\mathrm{P}$, Thant KZ, Sharma P. The genetics of sporadic ruptured and unruptured intracranial aneurysms: a genetic meta-analysis of 8 genes and 13 polymorphisms in approximately 20,000 individuals. J Neurosurg (2010) 112:714-21. doi:10.3171/2009.8.JNS092

8. Lad SP, Hegen H, Gupta G, Deisenhammer F, Steinberg GK. Proteomic biomarker discovery in cerebrospinal fluid for cerebral vasospasm following subarachnoid hemorrhage. J Stroke Cerebrovasc Dis (2012) 21:30-41. doi:10.1016/ j.jstrokecerebrovasdis.2010.04.004

9. Arati S, Sibin MK, Bhat DI, Narasingarao KV, Chetan GK. Polymorphisms of apolipoprotein E and aneurysmal subarachnoid haemorrhage: a metaanalysis. Meta Gene (2016) 9:151-8. doi:10.1016/j.mgene.2016.06.003

10. Mahley RW. Apolipoprotein E: cholesterol transport protein with expanding role in cell biology. Science (1988) 240:622-30. doi:10.1126/science.3283935

11. Gallek MJ, Conley YP, Sherwood PR, Horowitz MB, Kassam A, Alexander SA. APOE genotype and functional outcome following aneurysmal subarachnoid hemorrhage. Biol Res Nurs (2009) 10:205-12. doi:10.1177/1099800408323221

12. Juvela S, Siironen J, Lappalainen J. Apolipoprotein E genotype and outcome after aneurysmal subarachnoid hemorrhage. J Neurosurg (2009) 110:989-95. doi:10.3171/2008.11.JNS081266

13. D'ambrosio AL, Kreiter KT, Bush CA, Sciacca RR, Mayer SA, Solomon RA, et al. Far lateral suboccipital approach for the treatment of proximal posteroinferior cerebellar artery aneurysms: surgical results and long-term outcome. Neurosurgery (2004) 55:39-50; discussion 50-4. submitted version of manuscript: YJ, X-cS, and L-gC. Statistical analysis: YW, J-hD, and J-wP. Study supervision: YJ, X-cS, and L-gC.

\section{ACKNOWLEDGMENTS}

We are grateful to Ms. Xiao-cui Tian, Chongqing Key Laboratory of Biochemistry and Molecular Pharmacology at the Chongqing Medical University for statistical analysis and technical support.

\section{FUNDING}

This work was supported by the National Natural Science Foundation of China (81371319 and 81571159), the Program for New Century Excellent Talents in University (NCET-12-1057), and the Foundation for outstanding youth academic technology leaders of Sichuan province (2014JQ0022). Luzhou GovernmentSouthwest Medical University Strategic Cooperation Project (2016LZXNYD-J12).

14. Leira EC, Davis PH, Martin CO, Torner JC, Yoo B, Weeks JB, et al Improving prediction of outcome in "good grade" subarachnoid hemorrhage. Neurosurgery (2007) 61:470-3. doi:10.1227/01.NEU.0000290891.39330.05

15. Aaslid R, Huber P, Nornes H. Evaluation of cerebrovascular spasm with transcranial Doppler ultrasound. J Neurosurg (1984) 60:37-41. doi:10.3171/jns. 1984.60.1.0037

16. Jiang Y, Sun X, Xia Y, Tang W, Cao Y, Gu Y. Effect of APOE polymorphisms on early responses to traumatic brain injury. Neurosci Lett (2006) 408:155-8. doi:10.1016/j.neulet.2006.08.082

17. Speck V, Staykov D, Huttner HB, Sauer R, Schwab S, Bardutzky J. Lumbar catheter for monitoring of intracranial pressure in patients with posthemorrhagic communicating hydrocephalus. Neurocrit Care (2011) 14:208-15. doi:10.1007/s12028-010-9459-6

18. Lanterna LA, Ruigrok Y, Alexander S, Tang J, Biroli F, Dunn LT, et al. Meta-analysis of APOE genotype and subarachnoid hemorrhage: clinical outcome and delayed ischemia. Neurology (2007) 69:766-75. doi:10.1212/01. wnl.0000267640.03300.6b

19. Lin B, Dan W, Jiang L, Yin XH, Wu HT, Sun XC. Association of APOE polymorphism with the change of brain function in the early stage of aneurysmal subarachnoid hemorrhage. Acta Neurochir Suppl (2011) 110:39-42. doi:10.1007/978-3-7091-0353-1_7

20. Tang J, Zhao J, Zhao Y, Wang S, Chen B, Zeng W. Apolipoprotein E epsilon4 and the risk of unfavorable outcome after aneurysmal subarachnoid hemorrhage. Surg Neurol (2003) 60:391-6; discussion 396-397. doi:10.1016/S00903019(03)00323-9

21. Wu HT, Zhang XD, Su H, Jiang Y, Zhou S, Sun XC. Association of apolipoprotein E polymorphisms with cerebral vasospasm after spontaneous subarachnoid hemorrhage. Acta Neurochir Suppl (2011) 110:141-4. doi:10.1007/ 978-3-7091-0353-1_24

22. Yin C, Ruan J, Jiang L, Zhou S, Sun X. The relationship between rebleeding after spontaneous SAH and APOE polymorphisms in a Chinese population. Int J Neurosci (2012) 122:472-6. doi:10.3109/00207454.2012.678445

23. Nornes $H$. The role of intracranial pressure in the arrest of hemorrhage in patients with ruptured intracranial aneurysm. J Neurosurg (1973) 39:226-34. doi:10.3171/jns.1973.39.2.0226

24. Olivecrona M, Wildemyr Z, Koskinen LO. The apolipoprotein E epsilon4 allele and outcome in severe traumatic brain injury treated by an intracranial pressure-targeted therapy. J Neurosurg (2010) 112:1113-9. doi:10.3171/2009.8. JNS09636

25. Gao J, Wang H, Sheng H, Lynch JR, Warner DS, Durham L, et al. A novel apoE-derived therapeutic reduces vasospasm and improves outcome in a murine model of subarachnoid hemorrhage. Neurocrit Care (2006) 4:25-31. doi:10.1385/NCC:4:1:025 
26. Chen L, Sun X, Jiang Y, Kuai L. APOEe4 increases trauma induced early apoptosis via reducing delayed rectifier $\mathrm{K}(+)$ currents in neuronal/glial co-cultures model. Exp Cell Res (2015) 334:294-300. doi:10.1016/j.yexcr.2015.03.004

27. Grubb RL Jr, Raichle ME, Eichling JO, Gado MH. Effects of subarachnoid hemorrhage on cerebral blood volume, blood flow, and oxygen utilization in humans. J Neurosurg (1977) 46:446-53. doi:10.3171/jns.1977.46.4.0446

28. Vassilouthis J, Richardson AE. Ventricular dilatation and communicating hydrocephalus following spontaneous subarachnoid hemorrhage. J Neurosurg (1979) 51:341-51. doi:10.3171/jns.1979.51.3.0341

29. Rabinstein AA, Weigand S, Atkinson JL, Wijdicks EF. Patterns of cerebral infarction in aneurysmal subarachnoid hemorrhage. Stroke (2005) 36:992-7. doi:10.1161/01.STR.0000163090.59350.5a

30. Karnchanapandh K. Effect of increased intracranial pressure on cerebral vasospasm in SAH. Acta Neurochir Suppl (2008) 102:307-10. doi:10.1007/978-3211-85578-2_58

31. Karnchanapandh K. Effect of increased ICP and decreased CPP on DND and outcome in ASAH. Acta Neurochir Suppl (2012) 114:339-42. doi:10.1007/9783-7091-0956-4_65

32. Platz J, Guresir E, Wagner M, Seifert V, Konczalla J. Increased risk of delayed cerebral ischemia in subarachnoid hemorrhage patients with additional intracerebral hematoma. J Neurosurg (2017) 126(2):504-10. doi:10.3171/2015.12. JNS151563

33. Tai LM, Thomas R, Marottoli FM, Koster KP, Kanekiyo T, Morris AW, et al. The role of APOE in cerebrovascular dysfunction. Acta Neuropathol (2016) 131:709-23. doi:10.1007/s00401-016-1547-z

34. Howarth C. The contribution of astrocytes to the regulation of cerebral blood flow. Front Neurosci (2014) 8:103. doi:10.3389/fnins.2014.00103

35. Yeo SS, Choi BY, Chang CH, Kim SH, Jung YJ, Jang SH. Evidence of corticospinal tract injury at midbrain in patients with subarachnoid hemorrhage. Stroke (2012) 43:2239-41. doi:10.1161/STROKEAHA.112.661116

36. Egashira Y, Zhao H, Hua Y, Keep RF, Xi G. White matter injury after subarachnoid hemorrhage: role of blood-brain barrier disruption and matrix metalloproteinase-9. Stroke (2015) 46:2909-15. doi:10.1161/STROKEAHA. 115.010351

37. Jang SH, Choi BY, Kim SH, Chang CH, Jung YJ, Kwon HG. Injury of the mammillothalamic tract in patients with subarachnoid haemorrhage: a retrospective diffusion tensor imaging study. BMJ Open (2014) 4:e005613. doi:10.1136/ bmjopen-2014-005613

38. Kummer TT, Magnoni S, Macdonald CL, Dikranian K, Milner E, Sorrell J, et al. Experimental subarachnoid haemorrhage results in multifocal axonal injury. Brain (2015) 138:2608-18. doi:10.1093/brain/awv180

39. van Asch CJ, van der Schaaf IC, Rinkel GJ. Acute hydrocephalus and cerebral perfusion after aneurysmal subarachnoid hemorrhage. AJNR Am J Neuroradiol (2010) 31:67-70. doi:10.3174/ajnr.A1748
40. Tomimoto H, Ihara M, Wakita H, Ohtani R, Lin JX, Akiguchi I, et al. Chronic cerebral hypoperfusion induces white matter lesions and loss of oligodendroglia with DNA fragmentation in the rat. Acta Neuropathol (2003) 106:527-34. doi:10.1007/s00401-003-0749-3

41. Alosco ML, Brickman AM, Spitznagel MB, Griffith EY, Narkhede A, Cohen R, et al. Reduced cerebral blood flow and white matter hyperintensities predict poor sleep in heart failure. Behav Brain Funct (2013) 9:42. doi:10.1186/17449081-9-42

42. Barker R, Ashby EL, Wellington D, Barrow VM, Palmer JC, Kehoe PG, et al. Pathophysiology of white matter perfusion in Alzheimer's disease and vascular dementia. Brain (2014) 137:1524-32. doi:10.1093/brain/awu040

43. Pang J, Chen Y, Kuai L, Yang P, Peng J, Wu Y, et al. Inhibition of blood-brain barrier disruption by an apolipoprotein E-mimetic peptide ameliorates early brain injury in experimental subarachnoid hemorrhage. Transl Stroke Res (2016). doi:10.1007/s12975-016-0507-1

44. Lanterna LA, Rigoldi M, Tredici G, Biroli F, Cesana C, Gaini SM, et al. APOE influences vasospasm and cognition of noncomatose patients with subarachnoid hemorrhage. Neurology (2005) 64:1238-44. doi:10.1212/01.WNL. 0000156523.77347.B4

45. Soehle M, Chatfield DA, Czosnyka M, Kirkpatrick PJ. Predictive value of initial clinical status, intracranial pressure and transcranial Doppler pulsatility after subarachnoid haemorrhage. Acta Neurochir (Wien) (2007) 149:575-83. doi:10.1007/s00701-007-1149-6

46. Wu Y, Pang J, Peng J, Cao F, Vitek MP, Li F, et al. An apoE-derived mimic peptide, COG1410, alleviates early brain injury via reducing apoptosis and neuroinflammation in a mouse model of subarachnoid hemorrhage. Neurosci Lett (2016) 627:92-9. doi:10.1016/j.neulet.2016.05.058

47. Pang J, Wu Y, Peng J, Yang P, Kuai L, Qin X, et al. Potential implications of apolipoprotein $\mathrm{E}$ in early brain injury after experimental subarachnoid hemorrhage: involvement in the modulation of blood-brain barrier integrity. Oncotarget (2016) 7(35):56030-44. doi:10.18632/oncotarget.10821

Conflict of Interest Statement: The authors declare that the research was conducted in the absence of any commercial or financial relationships that could be construed as a potential conflict of interest.

Copyright (c) 2017 Peng, Qin, Pang, Wu, Dong, Huang, Wan, Yang, Sun, Chen and Jiang. This is an open-access article distributed under the terms of the Creative Commons Attribution License (CC BY). The use, distribution or reproduction in other forums is permitted, provided the original author(s) or licensor are credited and that the original publication in this journal is cited, in accordance with accepted academic practice. No use, distribution or reproduction is permitted which does not comply with these terms. 